\title{
Optimization of the Cascade Code Parameters by the Criterion of the Boundary Value of the Interference Band and the Selection of the Decoding Algorithm
}

Sergey B. Zhironkin*, Andrey A. Pshenitsyn and Alexander A. Bliznyuk VKO Military Academy named after Marshal of the Soviet Union Georgiy Zhukov Tver, Russian Federation

Received 12.03.2021, received in revised form 06.07.2021, accepted 15.08.2021

\begin{abstract}
A coherent data transmission channel with pseudo-random tuning of the operating frequency (FH) is considered, a concatenated code based on an ensemble of orthogonal signals and a Reed-Solomon code, with various reception-decoding algorithms under conditions of simultaneous action on the channel of two interference - a barrage and a FH concentrated in a part of the band.

The estimates of the boundary value of the part of the FH band covered by the interference, the characteristics of the optimal concatenated code, are obtained, and also the receive-decoding algorithm is selected, in which the boundary value of the part of the band covered by the interference takes the maximum value in a given interference environment.
\end{abstract}

Keywords: coherent data transmission channel, signal-to-noise ratio, concatenated coding, noise immunity, soft decoding, hard decoding.

Citation: Zhironkin S. B., Pshenitsyn A. A., Bliznyuk A. A. Optimization of the cascade code parameters by the criterion of the boundary value of the interference band and the selection of the decoding algorithm, J. Sib. Fed. Univ. Eng. \& Technol., 2021, 14(7), 820-829. DOI: 10.17516/1999-494X-0347

(C) Siberian Federal University. All rights reserved

This work is licensed under a Creative Commons Attribution-Non Commercial 4.0 International License (CC BY-NC 4.0).

* Corresponding author E-mail address: iadrin@mail.ru 


\title{
Оптимизация параметров каскадного кода
}

\section{по критерию максимума граничного значения полосы частот помехи и выбор алгоритма декодирования}

\author{
С. Б. Жиронкин, А. А. Пшеницын, А. А. Близнюк \\ Военная академия воздушно-космической оборонь \\ имени Маршала Советского Союза Г.К. Жукова \\ Российская Федеращия, Тверь
}

\begin{abstract}
Аннотация. Рассмотрен когерентный канал передачи данных с псевдослучайной перестройкой рабочей частоты (ППРЧ), каскадным кодом на основе ансамбля ортогональных сигналов и кода Рида-Соломона, с различными алгоритмами приема-декодирования в условиях одновременного действия на канал двух помех - заградительной и сосредоточенной в части полосы ППРЧ. Получены оценки граничного значения части полосы ППРЧ, накрытой помехой, характеристики оптимального каскадного кода, а также выбран алгоритм приема-декодирования, при котором граничное значение части полосы, накрытой помехой, принимает максимальное значение в заданной помеховой обстановке.
\end{abstract}

Ключевые слова: когерентный канал передачи данных, отношение сигнал/шум, каскадное кодирование, помехоустойчивость, мягкое декодирование, жесткое декодирование.

Цитирование: Жиронкин, С. Б. Оптимизация параметров каскадного кода по критерию максимума граничного значения полосы частот помехи и выбор алгоритма декодирования / С. Б. Жиронкин, А. А. Пшеницын, А. А. Близнюк // Журн. Сиб. федер. ун-та. Техника и технологии, 2021, 14(7). С. 820-829. DOI: 10.17516/1999-494X-0347

\section{Введение}

Для канала передачи данных с ППРЧ помеха в части рабочей полосы частот, занимаемой спектром сигналов, имеет импульсный характер. Для практики характерен случай, когда совместно с импульсной (узкополосной) помехой действует непрерывная (широкополосная) помеха [1-3]. Кроме того, доказано [4], что при ППРЧ с большим числом рабочих частот наихудшая помеха в каждый момент времени равномерно выбирает часть из них, на которую воздействует с одним превышением, а на оставшуюся часть - с другим. Такая помеха может быть представлена как смесь двух помех - заградительной и сосредоточенной в части полосы ППРЧ, определяемая $\rho=\Delta F_{\text {помехи }} / \Delta F_{\text {Ппрч. }}$

Известно, что для систем связи (СC) с ППРЧ и помехоустойчивым кодированием существует значение $\rho_{\text {гр }}[5,6]$ части полосы, накрытой помехой, характеризующееся тем, что при $\rho \leq \rho_{\text {гр }}$ независимо от мощности помехи обеспечивается заданная достоверность передачи информации.

В современных помехоустойчивых СС с ППРЧ полоса частот может составлять 250 МГц и более $[7,8]$. Для таких СС характерно воздействие помехи в части полосы ППРЧ, одним из определяющих параметров которой является $\rho_{\text {гр. }}$.

\section{Расчет граничного значения части полосы ППРЧ, накрытой помехой, при жестком декодировании}

В каналах передачи данных с ППРЧ часто используется каскадное кодирование, на внутренней ступени которого применяется ансамбль из М ортогональных сигналов (ортогональный код $\left.\left(n_{2}=\mathrm{M}, k_{2}=\log _{2} \mathrm{M}\right)\right)$, а на внешней - код Рида-Соломона $(\mathrm{PC})\left(n_{1}, k_{1}\right)$. 
При жестком декодировании вероятность ошибки в блоке РС кода определяется выражением [9]

$$
P_{e_{6 \pi}}=\sum_{i=t+1}^{n_{1}} \mathrm{C}_{n_{1}}^{i} P_{e}^{i}(\rho)\left(1-P_{e}^{i}(\rho)\right)^{n_{1}-i}
$$

где $t$ - количество исправляемых ошибок в блоке; $n_{1}$ - общее число недвоичных символов (импульсов) в блоке РС кода; $\mathrm{C}_{n_{1}}^{i}$ - число сочетаний из $n_{1}$ по $i ; P_{e}(\rho)$ - вероятность ошибочного приема символа (вероятность ошибочного различения ортогональных сигналов).

В свою очередь, вероятность ошибочного различения ортогональных сигналов при когерентном приеме в канале с ППРЧ и действием двух помех определяется соотношением [9]

$$
\begin{aligned}
& P_{e}(\rho)=\rho\left[1-\frac{1}{\sqrt{2 \pi}} \int_{-\infty}^{\infty} \exp \left[-\frac{1}{2}\left(x-\sqrt{\frac{2 m E_{b i t}}{n_{1}\left(\left(N_{j} / \rho\right)+N_{n}\right)}}\right)^{2}\right] \cdot \Phi^{M-1}(x) d x\right]+ \\
& +(1-\rho)\left[1-\frac{1}{\sqrt{2 \pi}} \int_{-\infty}^{\infty} \exp \left[-\frac{1}{2}\left(x-\sqrt{\frac{2 m E_{b i t}}{n_{1} N_{n}}}\right)^{2}\right] \cdot \Phi^{M-1}(x) d x\right],
\end{aligned}
$$

где М - объем ансамбля ортогональных сигналов, используемых для передачи каждого символа кодового слова; $m$ - количество информации, переносимое блоком кода (бит); $N_{j}$ - спектральная плотность мощности помехи (шума), сосредоточенной в части полосы ППРЧ; $N_{n}$ - спектральная плотность мощности помехи (шума) во всей полосе ППРЧ; $E_{b i t}-$ энергия сигнала, приходящаяся на один бит переданной информации; $\Phi(x)=\frac{1}{\sqrt{2 \pi}} \int_{-\infty}^{x} e^{-\frac{t^{2}}{2}} d t-$ интеграл
вероятности.

В формуле (2) первое слагаемое определяет зависимость $P_{e}$ от $N_{j}$ и $N_{n}$ (сигнал излучен на частотной позиции, на которой действуют две помехи), а второе - только от $N_{n}$ (сигнал излучен на частотной позиции, на которой лишь помеха во всей полосе). Для расчета $\rho_{\text {гр }}$ спектральная плотность мощности помехи, накрывающая часть полосы, $N_{j} \rightarrow \infty$. Тогда первое слагаемое в формуле (2) равно $\rho$. В результате уравнение (2) принимает вид

$$
P_{e}\left(\rho_{\text {гр }}\right)=\rho_{\text {гр }}+\left(1-\rho_{\text {гр }}\right)\left[1-\frac{1}{\sqrt{2 \pi}} \int_{-\infty}^{\infty} \exp \left[-\frac{1}{2}\left(x-\sqrt{\frac{2 m E_{\text {bit }}}{n_{1} N_{n}}}\right)^{2}\right] \cdot \Phi^{M-1}(x) d x\right] .
$$

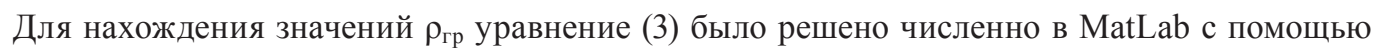
функции fzero. В результате расчетов для РC $\left(31, k_{1}\right)$ при вероятности ошибки $P_{e}\left(\rho_{\text {гр }}\right)$, соответ-

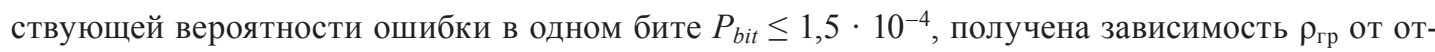
ношения сигнал/шум $E_{b i t} / N_{n}$ для различных РС кодов при $\mathrm{M}=32$ (рис. 1$)$.

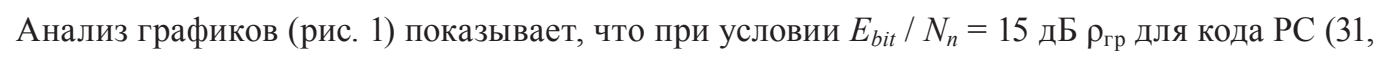

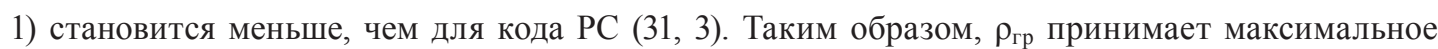

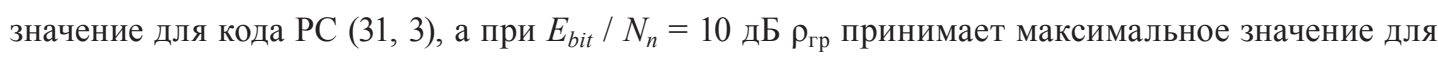
кода РС $(31,7)$. При заданном $n_{1}$ и отношении $E_{b i t} / N_{n}$ существует оптимальная скорость РС кода

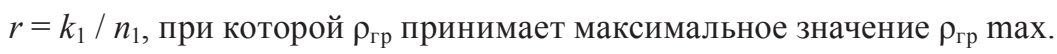




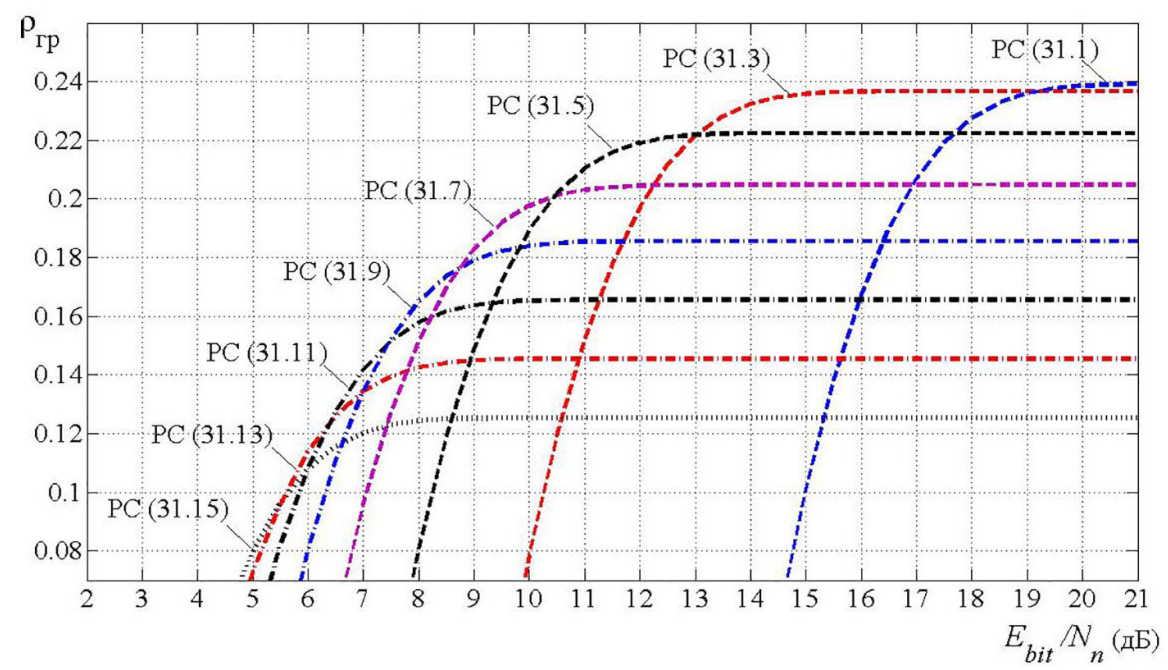

Рис. 1. Зависимость $\rho_{\text {гр }}$ от отношения сигнал/шум $E_{b i t} / N_{n}$

Fig. 1. Dependence of $\rho_{\text {гр }}$ on the signal-to-noise ratio $E_{b i t} / N_{n}$

\section{Оптимизация параметров каскадного кода}

\section{по критерию максимума граничного значения полосы частот помехи при мягком декодировании}

Для исследования зависимости $\rho_{\text {гр }} \max$ от $E_{b i t} / N_{n}$ и параметров каскадного кода в каналах с мягким декодированием и одновременным действием на канал двух помех расчетных соотношений нет, поэтому было произведено статистическое имитационное компьютерное моделирование.

Для реализации моделирования разработана математическая модель процесса когерентного приема-декодирования, в том числе мягкого, каскадного кода, и реализующая ее компьютерная программа в среде MatLab [10].

Непротиворечивость результатов моделирования была подтверждена тем, что полученные значения $\rho_{\text {гр }}^{*}$ для алгоритма жесткого декодирования отличаются не более чем на $5 \%$ от расчетных значений $\rho_{\text {гр }}$, полученных в результате численного решения уравнения (1).

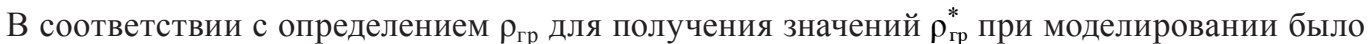
задано отношение сигнал/помеха $E_{b i t} / N_{n}=-20$ дБ (при моделировании $E_{b i t}=$ const).

Кроме помехи в части полосы ППРЧ в модели предусмотрено действие помехи (шума) во всей полосе частот СС, характеризующееся отношением сигнал/шум $E_{b i t} / N_{n}=15 ; 10 ; 5$ дБ. Моделирование производилось при заданной достоверности передачи информации, определяемой вероятностью ошибки на один бит передаваемой информации $P_{b i t} \leq 1,5 \cdot 10^{-4}$.

Результаты моделирования когерентного приема - мягкого декодирования по минимуму обобщенного расстояния (MOP) [11] для каскадных кодов (сигнально-кодовых конструкций СКК) на основе кодов РС $\left(15, k_{1}\right)$, PC $\left(31, k_{1}\right)$, РC $\left(63, k_{1}\right)$, PC $\left(127, k_{1}\right)$ приведены соответственно на рис. $2-4$.

По результатам анализа полученных графиков можно сделать следующие выводы:

1. Для каналов с исследованными СКК на интервале $0,68<r<1$, при $n_{1}-$ const, изменение $E_{b i t} / N_{n}$ практически не влияет на значение $\rho_{\text {гр }}^{*}$. 


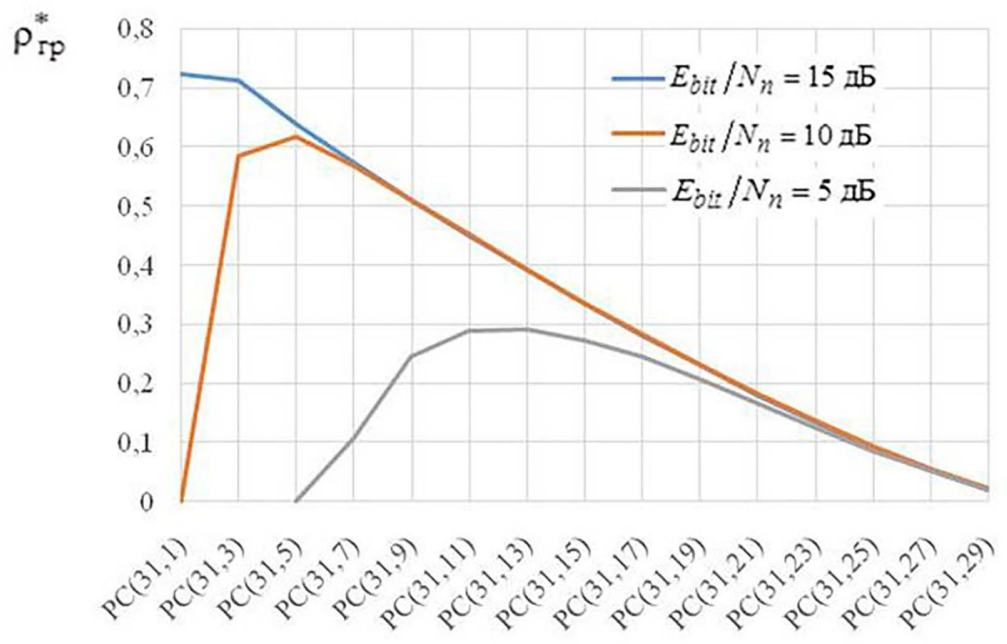

Рис. 2. Зависимость $\rho_{\text {гр }}^{*}$ от характеристик СКК при $k_{2}=5$

Fig. 2. Dependence of $\rho_{\text {гр }}^{*}$ on the characteristics of the SCC at $k_{2}=5$

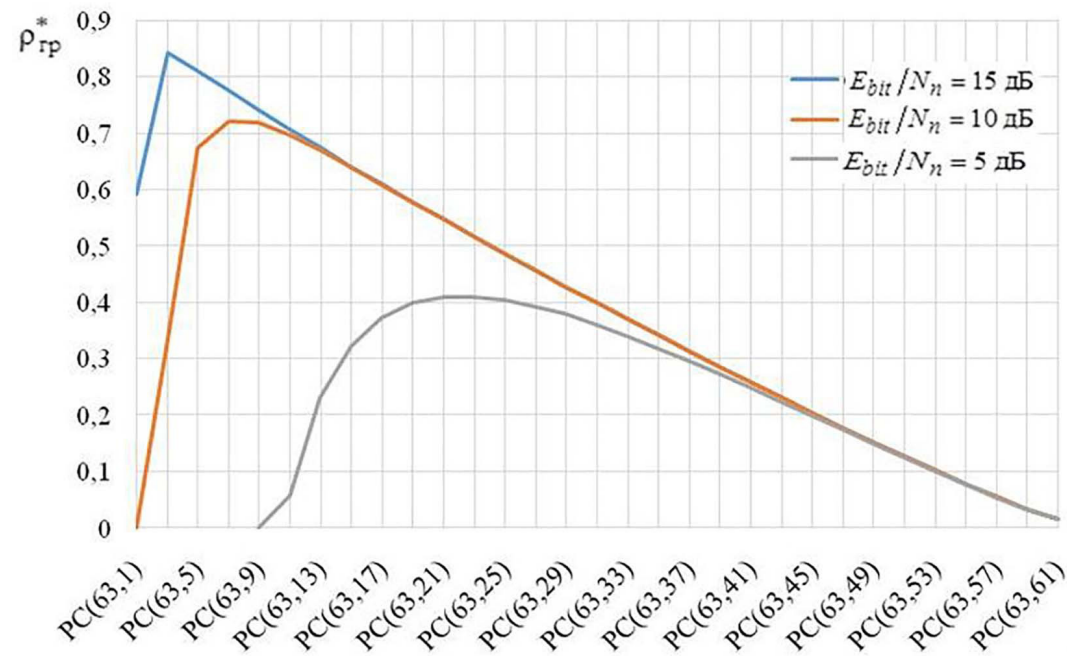

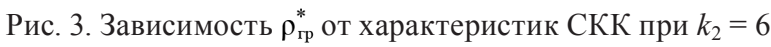

Fig. 3. Dependence of $\rho_{\mathrm{rp}}^{*}$ on the characteristics of the SCC at $k_{2}=6$

2. Для различных отношений $E_{b i t} / N_{n}$ и заданном $n_{1}$ существует максимальное значение $\rho_{\text {гр }}^{*} \operatorname{max,~которому~соответствует~оптимальная~скорость~РС~кода~} r$ : при $E_{b i t} / N_{n}=15$ дБ $-r \approx 0,03$; $E_{b i t} / N_{n}=10$ дБ $-r \approx 0,1 ; E_{b i t} / N_{n}=5$ дБ $-0,3<r<0,4$.

3. С уменьшением $E_{b i t} / N_{n}$ скорость РС кода $r$, при котором $\rho_{\text {гр }}^{*}$ тах, увеличивается.

4. При неизменном значении $E_{b i t} / N_{n}=$ const максимальное значение $\rho_{\text {гр }}^{*}$ max растет с увеличением $n_{1}$.

5. Для каждого значения отношения сигнал/помеха $E_{b i t} / N_{n}$ можно оптимизировать параметры РС кода таким образом, чтобы значения $\rho_{\text {гр }}^{*}$ были максимальными. 


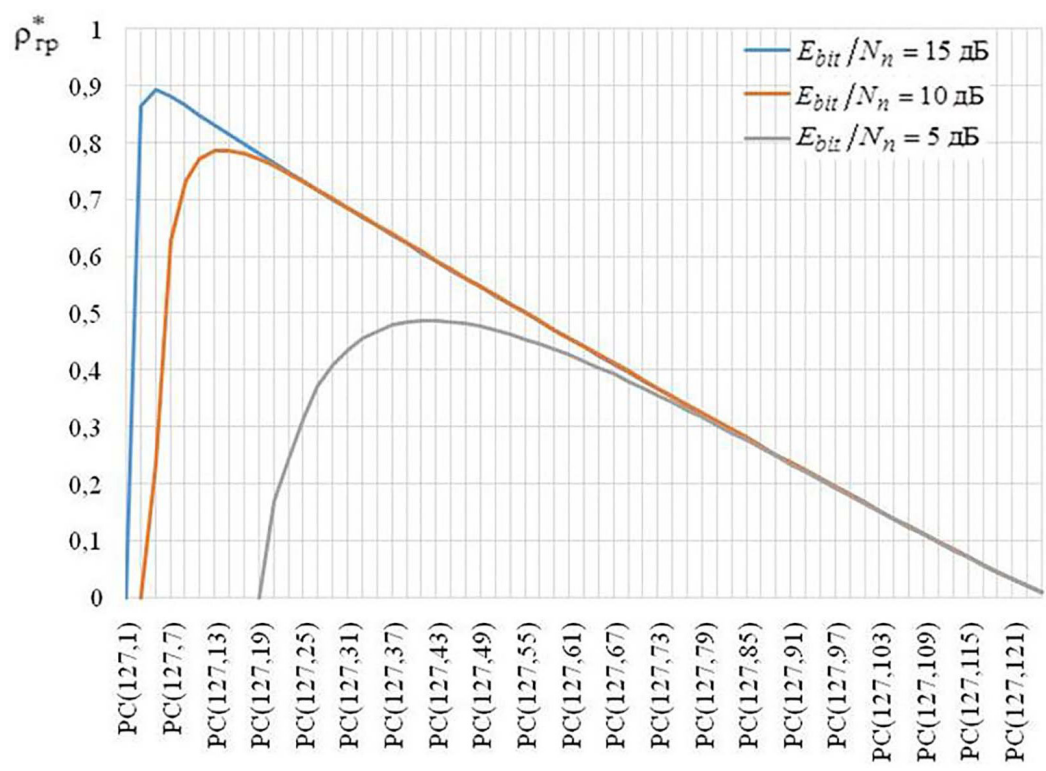

Рис. 4. Зависимость $\rho_{\text {гр }}^{*}$ от характеристик СКК при $k_{2}=7$

Fig. 4. Dependence of $\rho_{\mathrm{rp}}^{*}$ on the characteristics of the SCC at $k_{2}=7$

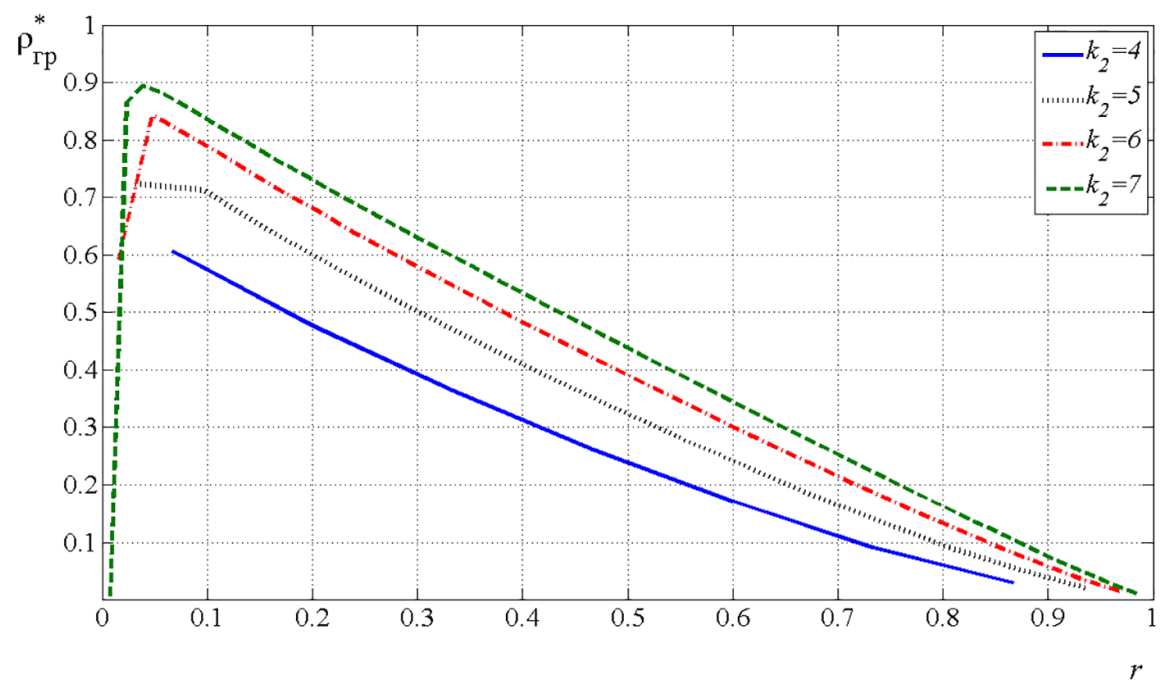

Рис. 5. Зависимость $\rho_{\text {гр }}^{*}$ от скорости кода $r$

Fig. 5. Dependence of $\rho_{\text {гр }}^{*}$ on the code rate $r$

Представляет интерес получение $\rho_{\text {гр }}^{*}$ для бо́льших значений $n_{1}=2^{k_{2}}-1$.

На рис. 5 представлены графики зависимости $\rho_{\text {гр }}^{*}$ от $r$ для $E_{b i t} / N_{n}=15$ дБ. При фиксированном значении $r$ можно предположить зависимость $\rho_{\text {гр }}^{*}$ от $k_{2}$. Программа MatLab позволяет аппроксимировать полученные данные. Наиболее точные результаты дает алгоритм аппроксимации Rational Trust-Region (MATLAB R2013b 8.2.0.701), определяемый выражением 


$$
f\left(k_{2}\right)=\frac{p_{1} k_{2}+p_{2}}{k_{2}+q_{1}}
$$

где $p_{1}, p_{2}, q_{1}$ - коэффициенты аппроксимации. При $r=0,2$ коэффициенты принимают значения $p_{1}=1,01 ; p_{2}=2,471 ; q_{1}=0,7228$. Увеличение количества отсчетов, участвующих в аппроксимации, повышает точность экстраполяции (табл. 1).

Таблица 1. Отклонение экстраполированных значений $\rho_{\text {гр }}^{*}$ от полученных в результате моделирования Table 1. Deviation of extrapolated $\rho_{\mathrm{rp}}^{*}$ values from those obtained as a result of modeling

\begin{tabular}{|c|c|c|c|}
\hline Участвуют & \multicolumn{3}{|c|}{ Экстраполированные (\%) } \\
\cline { 2 - 4 } в аппроксимации & $k_{2}=8$ & $k_{2}=9$ & $k_{2}=10$ \\
\hline$k_{2}=4 \ldots 7$ & 0,6 & 1,82 & 1,75 \\
\hline$k_{2}=4 \ldots 8$ & - & 1,29 & 1,03 \\
\hline$k_{2}=4 \ldots 9$ & - & - & 0,022 \\
\hline
\end{tabular}

\section{Выбор алгоритма декодирования}

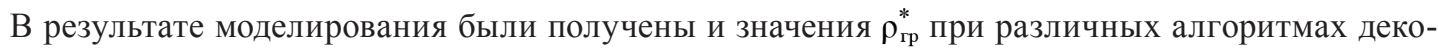
дирования, в том числе и мягких. В качестве мягких алгоритмов использовались: алгоритм со стиранием наименее надежных $l=n-k-2 t$ символов и исправлении до $t$ ошибок в оставшихся [12]; алгоритм Бородина [13], основанный на стирании наименее надежных $l=n-k$ символов $(t=0)$; алгоритм МОР $(t=0) \ldots(n-k)$, усеченный МОР - алгоритм [14], в котором реализуются первые три попытки алгоритма МОР $(t=0 \ldots 2)$.

При моделировании алгоритмов мягкого декодирования расчет апостериорной вероятности, определяющей наиболее надежные символы, производился с использованием известной (заданной) суммарной спектральной плотности мощности помехи (шума). Результаты моделирования для СКК на основе РС $(31, k)$ и $E_{b i t} / N_{n}=15 ; 10 ; 5$ дБ представлены на рис. 6-8.

Полученные графики показывают, что величина $\rho_{\text {гр }}^{*} \max$ принимает наибольшее значение при $E_{b i t} / N_{n}=15$ дБ и декодировании по алгоритмам: Бородина, МОР и усеченный МОР. С уменьшением $E_{b i t} / N_{n}$ до 5 дБ эффективность алгоритма Бородина по $\rho_{\text {гр }}^{*}$ уменьшается. При декодировании по алгоритмам МОР и усеченный МОР величина $\rho_{\text {гр }}^{*}$ max принимает наибольшее значение.

\section{Заключение}

1. За счет оптимизации параметров каскадного кода можно обеспечить граничное для помехи значение $\rho_{\text {гр }}^{*}$ части полосы частот канала с ППРЧ, превышающее 0,89 . Это означает, что для достижения заданного помехового эффекта противник должен ставить помеху, накрывающую не менее 89 \% полосы ППРЧ.

2. За счет выбора алгоритма декодирования можно обеспечить увеличение $\rho_{\text {гр в }}^{*} 2,5$ раза и более при переходе от алгоритма жесткого декодирования к оптимизированному алгоритму мягкого декодирования.

3. При увеличении интенсивности помехи во всей полосе $\rho_{\text {гр }}^{*}$ max уменьшается.

$$
-826-
$$




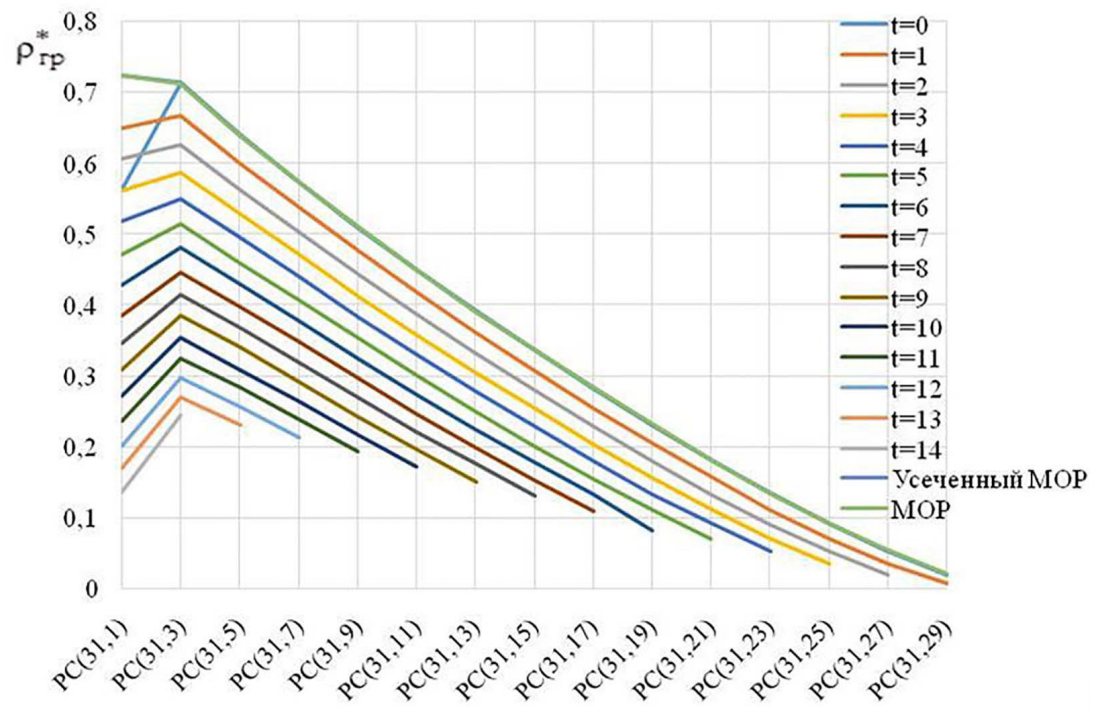

Рис. 6. Зависимость $\rho_{\text {гр }}^{*}$ от характеристик СКК и алгоритма приема-декодирования при $E_{b i t} / N_{n}=15$ дБ

Fig. 6. Dependence of $\rho_{\mathrm{rp}}^{*}$ on the characteristics of the SCC and the receive-decoding algorithm at $E_{b i t} / N_{n}=15$ дБ

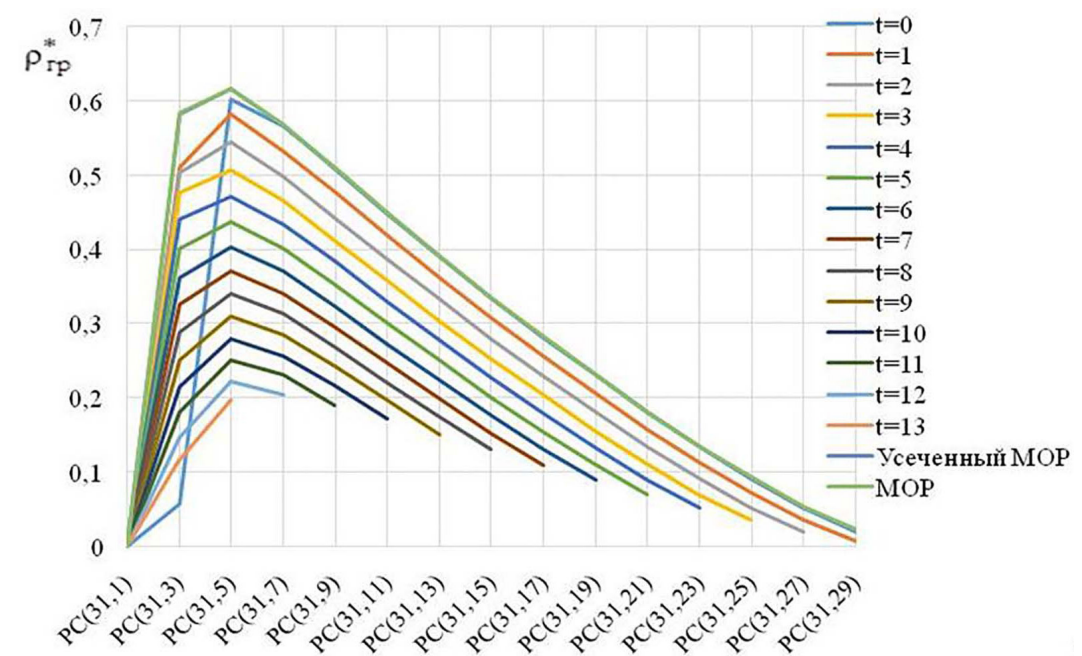

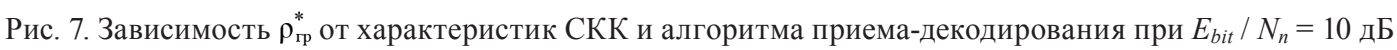

Fig. 7. Dependence of $\rho_{\text {гр }}^{*}$ on the characteristics of the SCC and the receive-decoding algorithm at $E_{b i t} / N_{n}=10$ дБ

Таким образом, полученные результаты позволяют выбрать параметры СКК, а также ал-

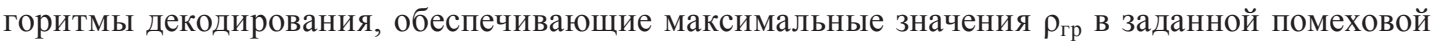
обстановке. 


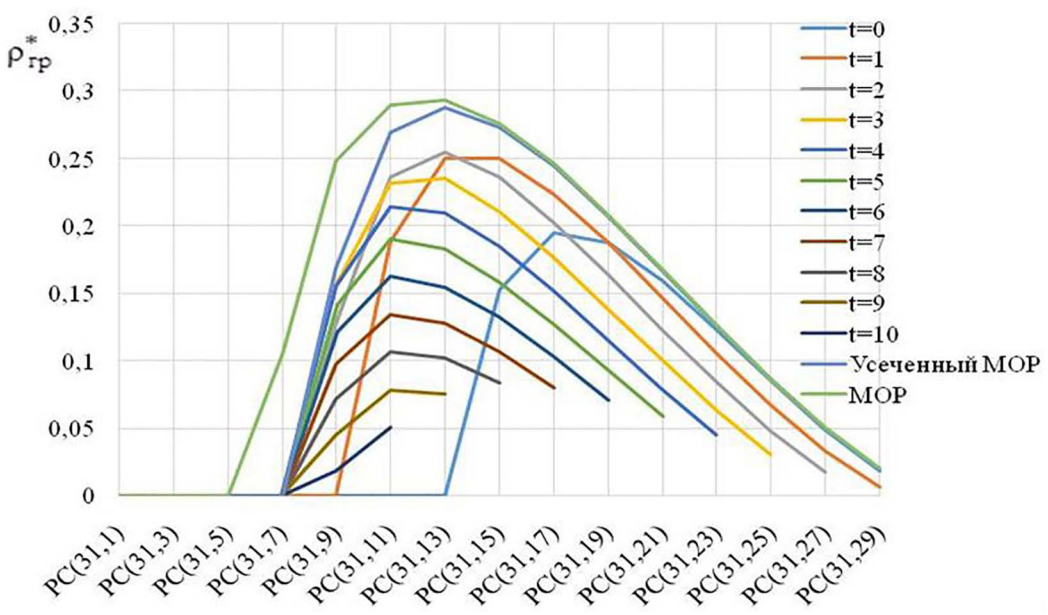

Рис. 8. Зависимость $\rho_{\text {гр }}^{*}$ от характеристик СКК и алгоритма приема-декодирования при $E_{b i t} / N_{n}=5$ дБ

Fig. 8. Dependence of $\rho_{\text {гр }}^{*}$ on the characteristics of the SCC and the receive-decoding algorithm at $E_{b i t} / N_{n}=5$ дБ

\section{Список литературы / References}

[1] Тузов Г.И. Помехозащищенность радиосистем со сложными сигналами: под ред. Г.И. Тузова. М: Радио и связь, 1985 [Tuzov G.I. Interference immunity of radio systems with complex signals: ed. G. I. Tuzov, Moskva, Radio i svyaz, 1985 (in Russian)].

[2] Зеленевский В.В., Зеленевский А.В., Джелаухян А.Ю. Методика оценки помехоустойчивости систем радиосвязи с фазоманипулированными сигналами с расширенным спектром при воздействии заградительных и сосредоточенных по полосе помех. Новые информационные технологии в системах связи и управления: труды XV Российской научно-техн. конф. Калуга: Ноосфера, 2016, 37-40 [Zelenevskij V.V., Zelenevskij A.V., Dzhelauhjan A. Ju. A technique for evaluating the noise immunity of radio communication systems with phase-shift keyed signals with a spread spectrum when exposed to barrage and band-centered interference. Novye informacionnye tehnologii $\mathrm{v}$ sistemah svjazi i upravlenija: trudy XV Rossijskoj nauchno-tehn. konf. Kaluga: Noosfera, 2016, 37-40 (in Russian)].

[3] Близнюк А.А., Жиронкин С.Б., Макарычев А. В. Энергетический подход к оценке помехоустойчивости канала передачи данных с псевдослучайной перестройкой рабочей частоты в условиях одновременного действия двух помех. Успехи современной радиоэлектроники. 2018, 12, 13-18 [Bliznyuk A. A., Zhironkin S. B., Makarychev A. V. Power approach to an assessment of a noise stability of a data link with pseudo-casual reorganization of working frequency in the conditions of simultaneous action of two hindrances. Achievements of Modern Radioelectronik, 2018, 12, 13-18 (in Russian)].

[4] Чуднов А.М. Помехозащищенность системы передачи информации с псевдослучайным переключением частот в условиях наихудших помех. Известия вузов. Радиоэлектроника. 1984, 9, 3-8 [Chudnov A.M. Interference immunity of the information transmission system with pseudo-random frequency switching in the conditions of the worst interference. Proceedings of universities. Radio electronics, 1984, 9, 3-8 (in Russian)]

[5] Pursley M. B., Stark W.E. Antijam capability of frequency-hop spread-spectrum with ReedSolomon coding, IEEE Military Communications Conference, 1983, pp.7-11. 
[6] Бердышев В.П., Близнюк А.А., Ж Жиронкин С. Б., Пшеницын А.А. Помехоустойчивость канала передачи данных с ППРЧ при мягком декодировании с использованием оценок спектральной плотности мощности помехи. Журнал Сибирского федерального университета. Техника и технологии. 2020, 13(7), 343-354 [Berdyshev V.P., Bliznyuk A. A., Zhironkin S. B., Pshenicyn A. A. Noise immunity of a data transmission channel with frequency hopping during soft decoding using estimates of the spectral density of the interference power. Journal of the Siberian Federal University. Technics and technology, 2020, 13(7), 343-354 (in Russian)]

[7] Скляр Б. Цифровая связь. Теоретические основы и практическое применение: пер. с англ. под ред. А.В. Назаренко. М.: Издательский дом «Вильямс», 2004 [Sklyar B. Digital communication. Theoretical foundations and practical application: trans, from English: ed. A. V. Nazarenko., Moskva, Izdatelskiy dom «Vilyams», 2004 (in Russian)]

[8] Борисов В.И., Чаркин Д.Ю. Архитектура и средства радиосвязи зарубежных комплексов управления. Теория и техника радиосвязи. 2016, 3, 5-17 [Borisov V.I., Charkin D. Yu. Architecture and means of radio communication of foreign control complexes. Theory and technology of radio communication. 2016, 3, 5-17 (in Russian)].

[9] Жиронкин С.Б., Близнюк А.А., Кучин А.А. Помехоустойчивость запросного канала опознавания с широкополосными сигналами и корректирующими кодами в условиях импульсных шумовых и внутрисистемных помех. Журнал Сибирского федерального университета «Техника и технологии», 2019, 12 (6), 673-682 [Zhhonkin S. B., Bliznuk A.A., Kuchin A.А. A noise stability of the request channel of an identification with broadband signals and correcting codes in the conditions of pulse noise and intersystem hindrances. Journal of Siberian Federal University «Engineering and technologies», 2019, 12 (6), 673-682 (in Russian)].

[10] Близнюк А. А., Мин Т. А., Пшеницын А. А. Программа исследования помехоустойчивости радиоканалов автоматической системы обмена данными с мягким декодером кода РидаСоломона. Свид. о гос. per. прогр. для ЭВМ 2017617335. Российская Федерация, заявл. 2017611424 от 20.02.17, опубл. 04.07.17. [Bliznyuk A. A., Min T. A., Pshenicyn A. A. Program of research of noise immunity of radio channels of an automatic data exchange system with a soft decoder of the ReedSolomon code. wit. about the state, per. prog, for computer 2017617335, Russian Federation, app. 2017611424 on 02/20/17, publ. 07/04/17 (in Russian)].

[11] Форни Д. Каскадные коды, пер. с англ, под ред. СИ Самойленко. М.: Мир, 1970 [Forni D. Concatenated codes: per. from English, ed. S. I. Samoilenko. Moskow, Mir, 1970 (in Russian)].

[12] Долгов В.И., Халимов Г.З. Декодирование блочных кодов со стиранием наименее надежных символов. Радиотехника. 1987, 3, 8-16 [Dolgov V.I. Halimov G.Z. Decoding of block codes with erasure of the least reliable symbols. Radio engineering. 1987, 3, 8-6 (in Russian)].

[13] Хворостенко Н.П. Статистическая теория демодуляиии дискретных сигналов. М.: Связь, 1968 [Hvorostenko N. P. Statistical theory of demodulation of discrete signals. Moskow, Svyaz, 1968 (in Russian)].

[14] Ковалев С.И. Два класса алгоритмов декодирования по минимуму обобщенного расстояния. Проблемы передачи информачии. 1986, XXII, 3, 41-54 [Kovalev S.I. Two Classes of Generalized Distance Minimum Decoding Algorithms. Information transfer problems. 1986, XXII, 3, 41-54 (in Russian)]. 